\title{
Neonatal Skin Disorders and the Emergency Medicine Physician
}

\author{
Gomathy Sethuraman, MD, ${ }^{*}$ Anthony J. Mancini, MD* $\dagger \dagger$
}

\begin{abstract}
Neonatal skin may play host to a variety of dermatological conditions, ranging in spectrum from benign, self-limited disorders to severe and/or life-threatening disease. Neonatal skin disorders may be of concern to parents and physicians alike, and may initially be evaluated in the urgent care clinic or emergency department, where specialty consultation may not always be readily available. In this paper, several neonatal skin conditions are briefly reviewed, including vesiculopustular disorders; those presenting with bullae, erosions and ulcerations; vascular and pigmented birthmarks; and disorders which present with skin erythema and scaling. This brief discussion is intended as a starting point for the emergency physician who may be the "front line" clinician faced with the evaluation of a neonate or infant with skin disease.
\end{abstract}

Clin Ped Emerg Med 9:200-209 (c) 2008 Elsevier Inc. All rights reserved.

KEYWORDS neonatal, skin disorders, emergency medicine

$\mathrm{T}$ he skin of the neonate may exhibit several differences from that of the adult, both anatomically and physiologically, and may play host to a variety of dermatological conditions, representing a wide array of severities from mild and self-limited to severe and/or lifethreatening. These conditions may include (but are not limited to) transient or physiologic phenomena, findings related to perinatal or obstetrical trauma, disorders of pigmentation, infectious diseases, inflammatory conditions, genetic disorders, and malignancies.

Skin disorders in the infant may be a source for concern to parents and physicians alike and may present for initial evaluation to the pediatric clinic, urgent care, or emergency department. Practitioners in these settings should be familiar with neonatal skin disorders, as immediate specialty consultation may not always be readily available. What follows is a brief discussion of several select neonatal skin disorders. The reader is referred to other sources for a more comprehensive review of these and other conditions.

\section{Types of Neonatal Skin Lesions}

Although there is no widely accepted and consistent classification, skin lesions in neonates may present as papules, plaques, patches, pustules, vesicles, bullae, erosions, or ulcerations. The most common presentation is that of vesiculopustular lesions, which can be the presenting feature for a variety of infectious, inflammatory, genetic, and transient neonatal disorders (Table 1) [1,2]. Bullae, erosions, and ulcerations may be caused by several disorders listed in Table 1, as well as staphylococcal scalded skin syndrome, congenital syphilis, epidermolysis bullosa, mastocytosis, bullous forms of ichthyosis, autoimmune blistering disease, aplasia cutis congenita, and several even less common conditions [1,2]. Some distinct categories of neonatal skin lesions include pigmented (ie, melanocytic nevus, Mongolian spots, nevus of Ota, café au lait macules) and vascular (ie, infantile hemangioma, port wine stain) birthmarks, and those presenting with erythema and scaling (Table 2). Several disorders from each of these categories are briefly discussed here.

*Division of Pediatric Dermatology, Children's Memorial Hospital, Chicago, IL.

$\dagger$ Department of Pediatrics, Northwestern University Feinberg School of Medicine, Chicago, IL.

$\ddagger$ Department of Dermatology, Northwestern University Feinberg School of Medicine, Chicago, IL.

Reprint requests and correspondence: Anthony J. Mancini, MD, Division of Pediatric Dermatology, 2300 Children’s Plaza \#107, Chicago, IL 60614. (E-mail: amancini@northwestern.edu) 
Table 1 Causes of vesiculopustular skin eruptions in neonates.

\begin{tabular}{l} 
Noninfectious \\
Erythema toxicum neonatorum \\
Miliaria (prickly heat) \\
Neonatal acne \\
Eosinophilic pustular folliculitis \\
Acropustulosis of infancy \\
Transient neonatal pustular melanosis \\
Langerhans cell histiocytosis \\
Incontinentia pigmenti \\
Infectious \\
Bacterial \\
Staphylococcus aureus \\
Streptococcus pyogenes/other streptococci \\
Pseudomonas aeruginosa \\
Listeria monocytogenes \\
Aspergillus infection \\
Viral \\
Herpes simplex virus \\
Varicella zoster virus \\
Cytomegalovirus \\
Fungal \\
Candida albicans \\
Infestations \\
Scabies (Sarcoptes scabiei) infestation \\
\hline
\end{tabular}

Adapted from Curr Opin Pediatr. 1997;9:396-405 and Gilliam et al [2].

\section{Vesiculopustular Disorders}

\section{Erythema Toxicum Neonatorum}

Erythema toxicum neonatorum is the most common cause of vesiculopustular lesions in neonates [3-5]. This benign, self-limited condition is usually seen in full-term infants and appears 24 to 72 hours after birth, although it occasionally exhibits a delayed onset. Erythema toxicum neonatorum presents as erythematous macules, wheals, papules, and pustules, which may involve the face, trunk, and proximal extremities (Figure 1). The palms and soles are usually spared. The blotchy erythema commonly waxes and wanes, similar to an urticarial eruption. Erythema toxicum neonatorum is diagnosed on a clinical basis, but if there is a question, a smear of pustular contents can be

Table 2 Conditions associated with neonatal skin erythema and scaling.

Seborrheic dermatitis

Atopic dermatitis

Diaper dermatitis

Nutritional/metabolic disorders

Ectodermal dysplasia

Immunodeficiency

Collodian baby/ichthyoses

Psoriasis

Neonatal lupus erythematosus

Congenital/neonatal candidiasis

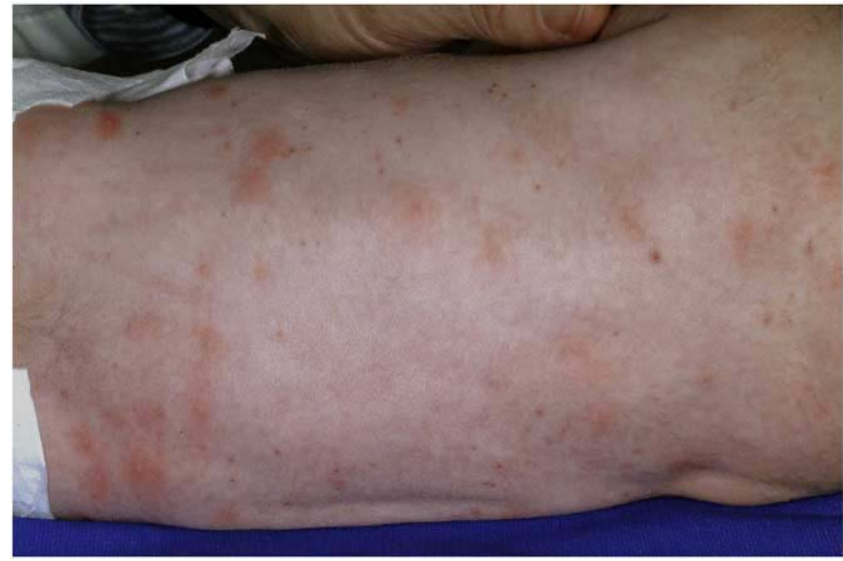

Figure 1 Erythema toxicum neonatorum. Blotchy erythematous wheals, papules, and papulopustules in a newborn.

performed and usually reveals abundant eosinophils. Peripheral eosinophilia may be seen in $15 \%$ to $18 \%$ of patients [5]. Erythema toxicum neonatorum usually resolves spontaneously over several days, and no treatment is required.

\section{Miliaria}

Miliaria is commonly seen during the first few weeks of life and is due to eccrine (sweat) duct obstruction. Predisposing factors include excessive warming in incubators, fever, warm clothing, and overswaddling. Two types of miliaria can occur in neonates, depending on the level of obstruction. In miliaria crystallina, the obstruction is very superficial, at the level of stratum corneum, which results in accumulation of sweat beneath it. Clinically, it presents as fragile, tiny, superficial, clear vesicles resembling water droplets that can easily be wiped away. Miliaria rubra, more commonly known as "prickly heat" and representing the most common form of miliaria, is due to obstruction of the sweat duct at the mid epidermis. Clinically, tiny erythematous papules, vesicles or papulopustules are present. Although miliaria can occur anywhere on the body surface, it is most common on occluded surfaces and in intertriginous areas. Treatment for miliaria is supportive, with avoidance of overheating, tepid to cool baths, and use of air conditioning when feasible $[1,5]$.

\section{Neonatal Acne}

Neonatal acne is seen in up to $20 \%$ of newborns [1]. It begins during the first few weeks of life and is believed to be related to the effects of maternal hormones on the newborn sebaceous glands. Malassezia species have also been hypothesized as a possible cause, in which case the eruption has been termed neonatal cephalic pustulosis $[6,7]$. Neonatal acne presents with erythematous papules and pustules limited usually to the face. Comedones are characteristically absent. Mild cases do not require therapy, and the process usually resolves spontaneously by 3 to 


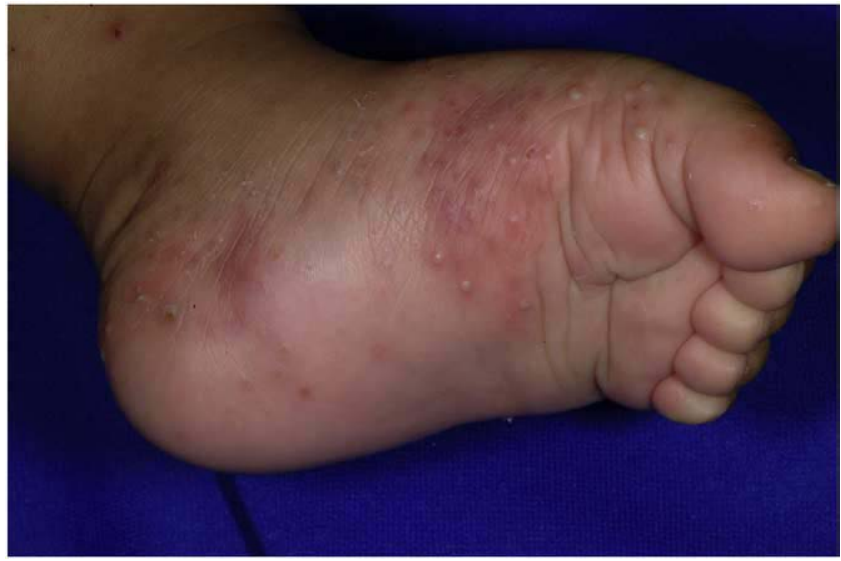

Figure 2 Infantile acropustulosis. Tense, pruritic pustules on the plantar and medial foot of a 3-month-old male.

6 months of age $[1,8]$. More severe cases may be treated with low strength (ie, $2.5 \%$ ) benzoyl peroxide gel or topical erythromycin. In patients where Malassezia is playing a pathogenic role, topical antifungal agents may speed resolution of the lesions [1].

\section{Acropustulosis of Infancy}

Acropustulosis of infancy (or infantile acropustulosis [IA]) is a pustular disorder characterized by recurrent crops of intensely pruritic, vesiculopustular lesions on the acral extremities. Lesions most often occur on the palms and soles (Figure 2), with occasional extension to dorsal surfaces as well as the wrists and ankles. Infantile acropustulosis can begin at any point between birth and the first year of life. Similar to eosinophilic pustular folliculitis, the lesions of IA tend to occur in crops and may wax and wane. However, pustular smears from patients with IA show mainly neutrophils, with occasional

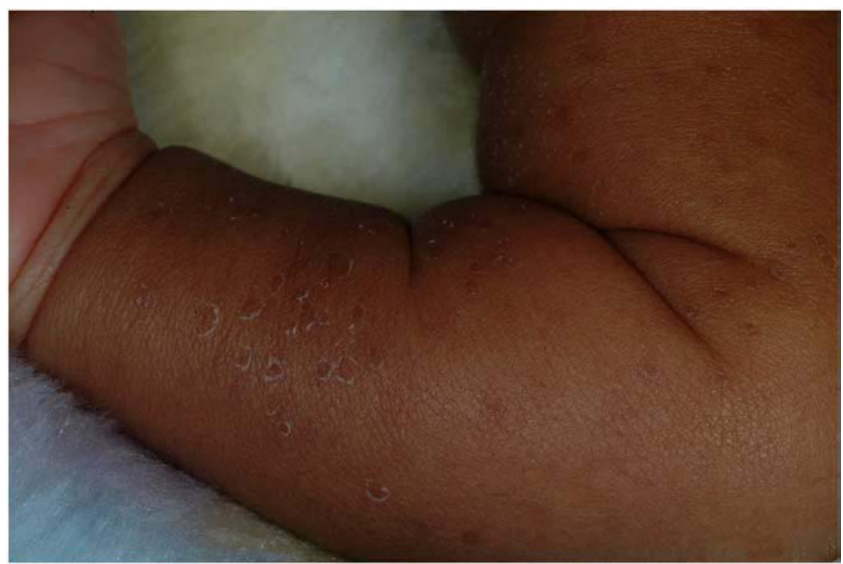

Figure 3 Transient neonatal pustular melanosis. Peripheral collarettes of scale mark areas of ruptured pustules in this newborn. Note the associated hyperpigmented macules. eosinophils. Although the etiology is unclear, a "postscabies" hypersensitivity phenomenon may be responsible of IA in some patients [9]. Treatment for IA includes use of antihistamines and potent topical corticosteroids during flare-ups. The flares of IA become less frequent and less intense until the process resolves completely, usually by 3 years of age [9].

\section{Transient Neonatal Pustular Melanosis}

Transient neonatal pustular melanosis (TNPM) is an uncommon benign neonatal skin disorder, seen primarily in black infants. It often presents in the immediate postnatal period and is characterized by vesiculopustules without associated erythema, which helps to distinguish it from more concerning vesiculopustular disorders such as herpes, varicella, Candida or staphylococcal/streptococcal infection. The pustules of TNPM (which contain primarily neutrophils) rupture easily, leaving behind hyperpigmented macules which may be surrounded by a characteristic collarette of scale (Figure 3) and may persist for several weeks to months. The most commonly affected sites include the forehead, chin, neck, upper chest, and back. The palms and soles may also be involved. Some infants with TNPM may present only with hyperpigmented macules, and it is assumed that the pustules ruptured in utero in these patients. No therapy is required $[1,5,8]$.

\section{Impetigo}

Impetigo is the most common bacterial skin infection in children, and it may occur in neonates as early as the first few days of life. It occurs in 2 forms: bullous and nonbullous $[10,11]$. Both Staphylococcus aureus and Streptococcus pyogenes can cause nonbullous impetigo, whereas most cases of bullous impetigo are caused by $S$ aureus. Nonbullous (also known as "crusted") impetigo presents with erythematous papules and vesicles with a

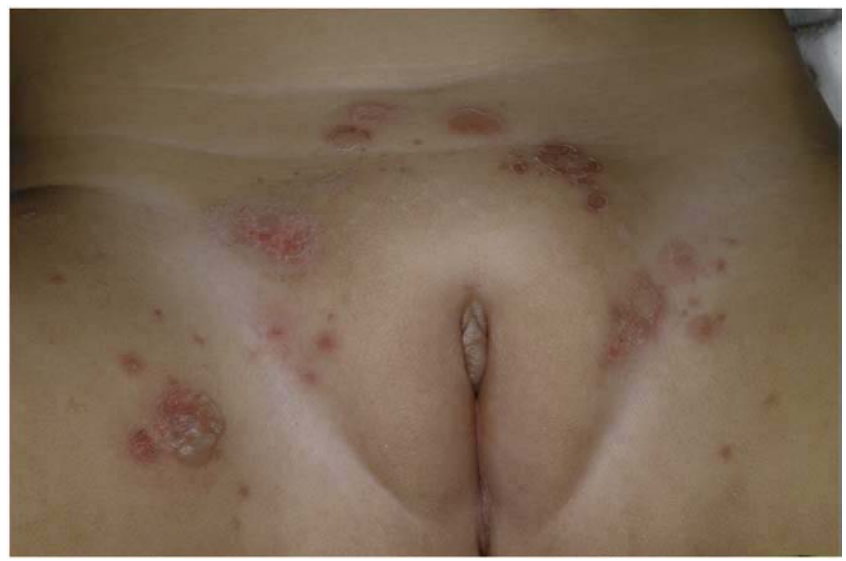

Figure 4 Bullous impetigo. Flaccid and ruptured bullae, with the characteristic peripheral collarette of the blister roof. 
honey-colored crust [10]. It is the lesser common form in neonates. Bullous impetigo, which can be considered a localized form of staphylococcal scalded skin syndrome (SSSS), is caused by infection with an epidermolytic toxinproducing strain of $S$ aureus. Clinically, it presents with fragile vesicles and bullae which rupture easily to form superficial erosive patches surrounded by a pathognomonic peripheral remnant of the blister roof (Figure 4) [12]. Common locations include the diaper, periumbilical, and intertriginous regions. A subset of bullous impetigo is "staphylococcal" pustulosis, which presents with fragile pustules with surrounding erythema that also rupture easily and leave a denuded erythematous macule with a peripheral rim of scale. Possible complications of neonatal impetigo include cellulitis, osteomyelitis, septic arthritis, pneumonia, and bacteremia. The diagnosis can be confirmed by Gram stain and culture, and treatment consists of an appropriate antimicrobial agent [10-12].

\section{Neonatal Herpes}

Neonatal herpes is one of the most potentially severe infections of the infant and occurs in 1 in 20000 to 1 in 3000 live births [13]. It is usually caused by herpes simplex virus type 2 and results from vertical transmission from an infected mother or via acquisition from passage through an infected birth canal [14]. Neonates can also acquire herpes infection postnatally by direct contact with infected persons. The risk of transmission of herpes to the newborn is higher with primary maternal genital infection (up to $50 \%$ transmission rate) than with recurrent infection (2\%$5 \%)[15,16]$.

Neonatal herpes occurs in 3 traditional forms: (i) skin, eyes, and mouth disease; (ii) central nervous system infection; and (iii) disseminated herpes. Skin, eyes, and mouth disease is seen in $40 \%$ of patients [17], although infants with this presentation may often progress to more disseminated involvement. Skin lesions may be present in roughly $77 \%$ and $60 \%$ of the infants with disseminated and central nervous system infection, respectively [18]. Neonatal herpes skin lesions present as erythematous macules and discrete or grouped vesicles on an erythematous base. The vesicles may transition to pustules over 1 to 2 days, and a more diffuse, vesiculobullous, and erosive dermatitis may also be noted in some patients. The lesions of neonatal herpes occur most commonly on the scalp and face and may also reveal a predominance in the region of the presenting part(s) [18].

Early recognition, prompt confirmation of diagnosis, and institution of therapy are crucial. Options for confirming the diagnosis from skin lesions include obtaining scrapings for a Tzanck smear, direct fluorescent antibody test, and/or viral culture. The direct fluorescent antibody, if available, is highly sensitive and specific and has the added advantage of a rapid turnaround (ie, hours) for results [19]. Viral cultures should also be obtained on blood, conjunctivae, nasopharynx, cerebrospinal fluid, and

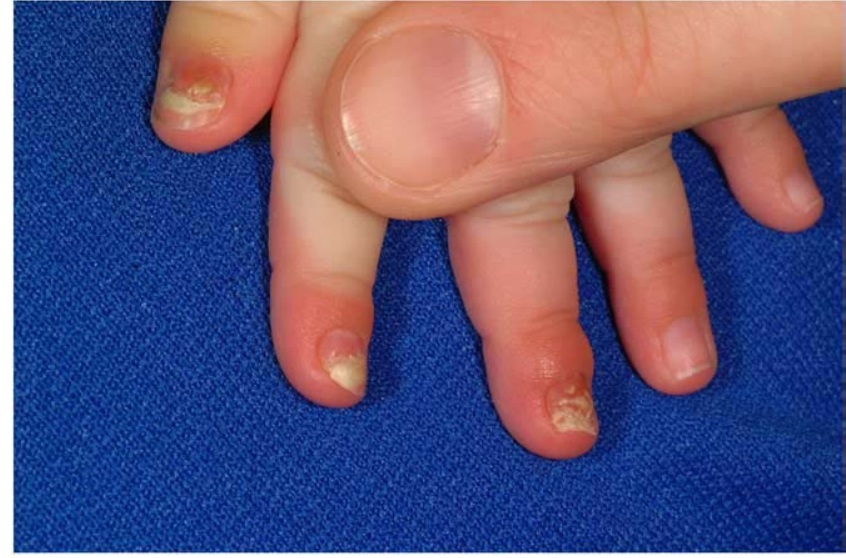

Figure 5 Congenital candidiasis of the nails. Yellow discoloration, ridging and periungal erythema were the only manifestations of congenital candidiasis in this newborn boy. Note the distal nail plate separation.

urine, and polymerase chain reaction studies of cerebrospinal fluid are now a standard component of the evaluation. High-dose parenteral acyclovir $(60 \mathrm{mg} / \mathrm{kg}$ per day) is the treatment of choice for neonatal herpes [20].

\section{Congenital and Neonatal Candidiasis}

In congenital candidiasis, lesions present between birth and 1 week of age, whereas in neonatal candidiasis, they present after 1 week of age. Congenital cutaneous candidiasis is acquired in utero and results from ascending infection with Candida albicans (and rarely other Candida species). Risk factors include premature labor, history of maternal vulvovaginitis, and presence of intrauterine foreign devices (such as cervical cerclage or intrauterine device). Invasive perinatal procedures and instrumentation, as well as extremely low birth weight, may predispose to more severe, disseminated forms of congenital candidiasis [21-23].

Congenital cutaneous candidiasis presents with scattered erythematous macules, papules, and papulopustules, which often number in the hundreds. Relative sparing of the diaper area is common, and oral thrush is rarely noted. Palm and sole involvement are commonly seen, and nail changes (yellow discoloration, ridging, and periungal erythema, Figure 5) are frequently present. Diffuse desquamation may eventually become more prominent, as the multiple pustules begin to rupture. Term infants without other risk factors tend to do well, often with infection limited to the skin $[8,24]$. However, in premature infants, dissemination is more likely. One notable population is extremely low-birth-weight infants, in whom widespread erosive changes and "burn-like" redness may occur in what has been termed "invasive fungal dermatitis" [25]. These infants are at high risk for invasive disease, including fungemia, urinary tract infection, and meningitis. 
The diagnosis of congenital candidiasis can be made with a bedside scraping and potassium hydroxide microscopic examination, which reveals budding spores and pseudohyphae. Fungal culture from intact pustules or other tissues can further confirm the diagnosis, and the findings of abscesses or funisitis on placental examination may also be useful. Topical antifungal agents often suffice in term infants with congenital cutaneous candidiasis and no risk factors for dissemination. Newborns with disseminated candidiasis or with known risk factors for disseminated disease require systemic antifungal therapy [26].

Neonatal candidiasis, which is seen after the first week of life, is usually acquired by passage through an infected maternal birth canal. It presents most often as oral thrush or diaper dermatitis but may also be associated with more severe or disseminated disease in newborns with other risk factors. Oral thrush is characterized by curd-like, white membranous patches on the tongue and oral mucous membranes. It can be confirmed by gentle scraping with a tongue blade, which reveals a friable erythematous mucosa underlying the removed patch [8]. Other vesiculopustular disorders which are not discussed here, and which may be seen in neonates, include EPF and neonatal scabies.

\section{Disorders Presenting With Bullae, Erosions, and Ulcerations}

\section{Staphylococcal Scalded Skin Syndrome}

Staphylococcal scalded skin syndrome is a toxin-mediated blistering disease caused by $S$ aureus [12,27-29]. The blisters are caused by epidermolytic toxins which separate the epidermal cells via specificity for the adhesion molecule desmoglein 1 [29]. Staphylococcal scalded skin syndrome begins with localized infection, usually around the umbilicus, perioral region (Figure 6A), conjunctivae, or perineum. Toxin is produced at the primary site of infection and then spreads in a hematogenous fashion, mediating the disease process at distant (nonprimary) sites. Clinically, it is characterized by widespread erythema associated with superficial fragile blisters (Figure 6B) and tender denudation. There is a predilection for the flexures, and radial crusting and fissuring around the mouth, nose, and ears may be noted. However, the oral mucous membranes are characteristically spared in SSSS. Accompanying symptoms include fever, irritability, decreased feeding, and lethargy [12].

The diagnosis of SSSS is often a clinical one, although the organism may be isolated in cultures of primary sites of cutaneous infection, the conjunctivae, the nasopharynx, or the blood. Although SSSS may be mild in older children, it tends to be a severe process in affected neonates and can be associated with significant morbidity or even mortality. Outbreaks of SSSS have been reported

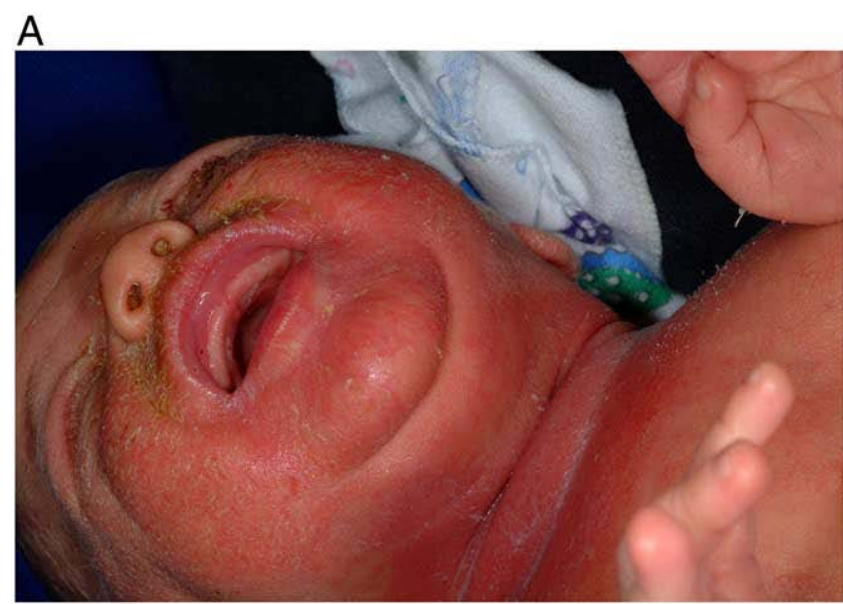

B

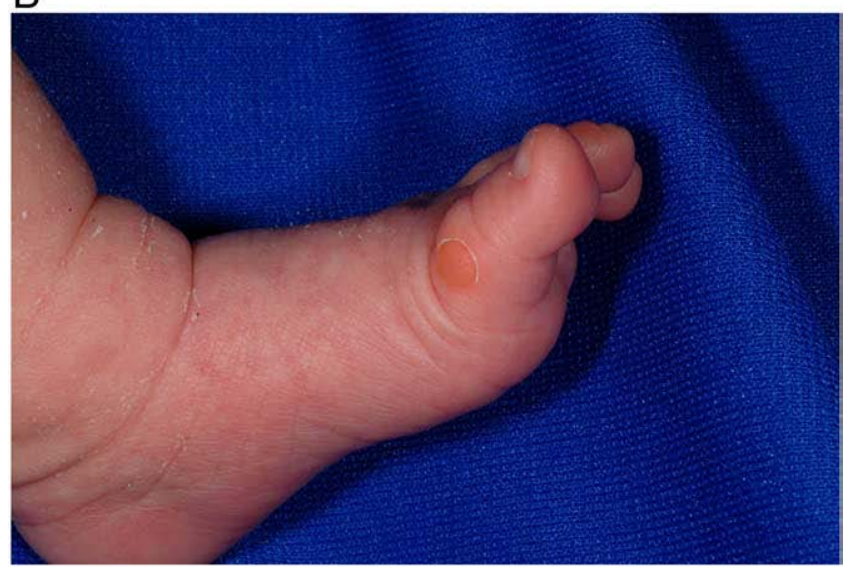

Figure 6 Staphylococcal scalded skin syndrome. This infant had initial infection characterized by perioral, perinasal and periorbital erythema with crusting (A). The ruptured, superficial blister on the toe (B) was one of the earliest manifestations of his generalized disease and is mediated by hematogenous spread of toxin.

in intensive care nurseries [30]. Treatment with a parenteral antistaphylococcal agent is indicated, along with meticulous wound care, pain control, and attention to fluid and electrolyte status.

\section{Epidermolysis Bullosa}

Epidermolysis bullosa (EB) is a group of inherited blistering diseases caused by mutations in different genes encoding the various structural proteins of the cutaneous basement membrane zone. The key feature in all forms of EB is the formation of blisters following abrasional skin trauma [31]. Depending on the level of blistering, EB is divided into 3 types: EB simplex ("epidermolytic EB"), junctional EB, and dystrophic (or "dermolytic") EB [31-35]. Neonates presenting with EB often have mucocutaneous blisters and erosions at the time of birth or shortly thereafter. Some may be born with large areas of denuded skin ("congenital localized 


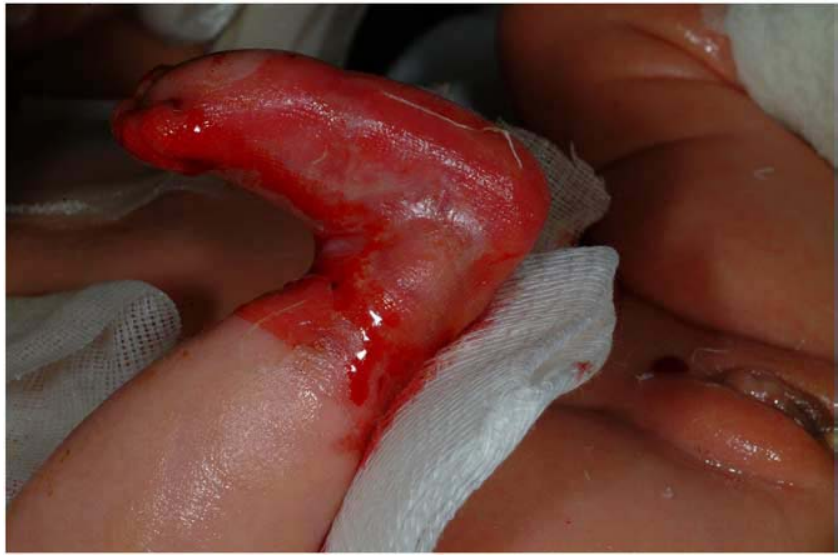

Figure 7 Epidermolysis bullosa. This newborn female presented with "congenital localized absence of skin." She was found to have recessive dystrophic epidermolysis bullosa, based on evaluation of skin biopsy with immunomapping studies.

absence of skin" or CLAS) (Figure 7). The subtype of EB cannot be confirmed based on clinical examination alone and requires studies such as skin biopsy with immunofluorescence mapping, electron microscopy, and/ or mutation analysis.

Epidermolysis bullosa simplex is the mildest and the most common form of EB. There are several different subtypes of this autosomal dominant disorder, including Weber-Cockayne (with blisters primarily occurring in an acral distribution), Koebner, and Dowling-Meara, among others. Weber Cockayne is the most common variant of EB simplex. In the more severe form, Dowling-Meara EB, the blisters are widespread and large, may occur in a grouped pattern (hence, the other name, "EB herpetiformis"), and may be associated with significant oral and esophageal mucosal involvement. Epidermolysis bullosa simplex lesions tend to heal without scarring, given the superficial plane of the blistering $[32,33]$.

Junctional EB is an autosomal recessive form which tends to be more severe. There are 3 major types, including the Herlitz form, the non-Herlitz form, and junctional EB with pyloric atresia. The Herlitz form of junctional EB is the most severe, with death often occurring by 2 years of age. Junctional EB often involves the nails, mucous membranes, and dental enamel. Affected sites often reveal atrophy and scarring, and growth retardation is common in those who survive the neonatal period [32].

Dystrophic EB can be either dominantly (dominant dystrophic EB) or recessively (recessive dystrophic EB) inherited. Both of these forms are associated with scarring and milia (tiny white cyst) formation. Dominant dystrophic EB is the more mild form, whereas recessive dystrophic EB is a severe variant characterized by widespread mucocutaneous disease, mitten-hand deformities, growth failure, recalcitrant anemia, esophageal stenosis, secondary infections, severe dental caries, and increased risk for squamous cell carcinoma [32]

Treatment for all forms of EB is primarily supportive, with attention to wound care, treatment of secondary infections, nutritional support, pain control, and psychologic support [36]. Prenatal diagnosis for subsequent pregnancies is available for all forms of EB [31].

\section{Vascular Birthmarks}

Vascular birthmarks are divided into vascular tumors and vascular malformations (Table 3). Vascular tumors are proliferative neoplasms of the vasculature, the most common of which is the infantile hemangioma. Vascular malformations represent developmental anomalies of blood vessels without any proliferative changes and may include capillary, venous, lymphatic and/or arterial elements [37,38]. Infantile hemangiomas and capillary malformations are briefly discussed here.

\section{Infantile Hemangiomas}

Infantile hemangiomas (IH) represent the most common benign skin/soft tissue tumor in children. They often begin to become evident during the first few weeks of life, although they may occasionally be congenital. They occur in superficial, deep, or mixed patterns. Superficial hemangiomas are bright red and grow out from the surface of the skin, whereas deep hemangiomas present as partially compressible nodules with an overlying bluish hue, venous prominence, and/or telangiectasia. In mixed lesions, both superficial and deep components are seen. Infantile hemangiomas undergo 3 phases in their natural history: the proliferative phase (from birth to approximately 10-12 months of age; this is the period of active growth), the plateau phase (a period of stability, usually occurring between 12 and 18 months of age), and the involution phase (spontaneous resolution, which occurs over the following 5-10 years). Most hemangiomas do not require therapy [37].

Congenital hemangiomas may represent a typical IH with early onset, or one of 2 distinct subtypes. The noninvoluting congenital hemangioma presents as a blue nodule or tumor with coarse surface telangiectasias and a surrounding

Table 3 The classification of vascular birthmarks.

\begin{tabular}{lc}
\hline Vascular tumors & Vascular malformations \\
\hline Infantile hemangioma & Capillary malformation \\
Tufted angioma & Nevus simplex \\
Kaposiform & (salmon patch) \\
hemangioendothelioma & Nevus flammeus \\
Pyogenic granuloma & (port wine stain) \\
Hemangiopericytoma & Venous malformation \\
& Lymphatic malformation \\
& Arteriovenous malformation \\
& Combined lesions \\
& Capillary-venous \\
& Capillary-lymphatic-venous
\end{tabular}




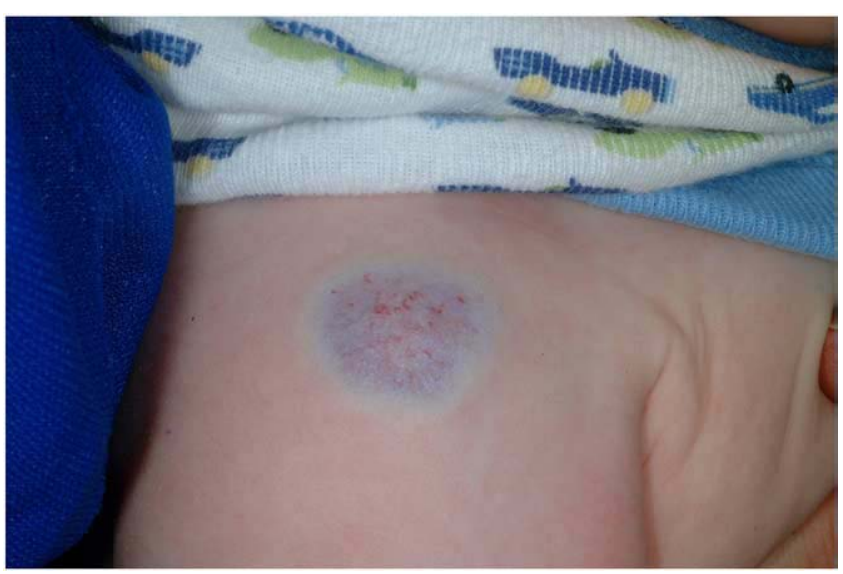

Figure 8 Non-involuting congenital hemangioma. This lesion is characterized by a blue hue, peripheral pallor, and coarse surface telangiectasias.

rim of pallor (Figure 8). These lesions persist indefinitely without spontaneous involution. The rapidly involuting congenital hemangiomas may present in a variety of patterns and is characterized by very rapid involution in early life, often with residual skin atrophy [37].

Infantile hemangiomas in certain locations may be associated with complications, including functional compromise, ulceration, bleeding, psychosocial concerns (later in life), and internal disease. Potentially concerning distribution patterns include the periocular, nasal tip, lip, anogenital, and ear regions. In addition, extensive and large (segmental) hemangiomas, those in a facial "beard" distribution, and large facial or lumbosacral lesions may have extracutaneous associations [37,38]. "PHACES" (Posterior fossa malformation, Hemangioma, Arterial anomalies, Cardiac defects and aortic Coarctation, Eye abnormalities, Sternal clefting and Supraumbilical abdominal raphe) syndrome (MIM 606519) refers to the constellation of extensive facial $\mathrm{IH}$ in association with defects in other systems, including the eyes, brain, heart, and arteries [39]. In addition, infants with multiple hemangiomas (ie, >5) are at risk for internal hemangiomatosis, most commonly involving the liver and gastrointestinal tract. Children with any of these forms of IH should be promptly referred to a specialist familiar with their evaluation and therapy $[37,38]$.

\section{Capillary Malformations}

Capillary malformations (CM) are the most common type of vascular malformation. The nevus simplex ("salmon patch") is, by far, the most common vascular lesion of infancy. These dull red pink macules and patches are most common on the glabella, superior eyelids, and inferior scalp. No treatment is necessary for these benign lesions, and most resolve spontaneously over several years. The exception is the inferior scalp CM ("stork bite"), which may persist indefinitely [40].
Nevus flammeus (or portwine stain [PWS]), is a congenital CM that presents as a pink or bright red vascular patch. These lesions may occur as an isolated skin finding or in association with a variety of syndromes. Portwine stain has a static course during the first few years but may darken progressively over a period of many years and rarely develops secondary proliferative blebs. Syndrome associations include SturgeWeber syndrome (V1 PWS in combination with glaucoma, leptomeningeal angiomatosis, and seizures), Klippel-Trenaunay syndrome (PWS, venous varicosities, and soft tissue/bony overgrowth; usually involves an extremity), Proteus syndrome (PWS, epidermal nevi, palmoplantar cerebriform hyperplasia, lipomas, macrodactyly, and hemimegalencephaly), and Cobb syndrome (dermatomal PWS with corresponding spinal cord vascular malformation) [40].

\section{Pigmented Birthmarks}

\section{Mongolian Spot}

Mongolian spots represent nevomelanocytes in the dermis, which underwent an arrest in migration from the neural crest to the epidermis. They are seen in most black infants and become proportionately less common with lighter skin types. They present as blue-to-gray patches, most often distributed over the buttocks and sacrum and occasionally more widespread. These benign lesions usually resolve spontaneously over the first few years of life. Extensive Mongolian spots have been reported in association with GM1 gangliosidosis type 1, Hunter syndrome, and Hurler syndrome $[41,42]$.

\section{Nevus of Ota}

Nevus of Ota is a periorbital dermal melanocytosis, which is congenital in $50 \%$ of the patients [3]. It presents as irregular, gray-to-blue macules and patches of the periorbital region, temple, forehead, and malar region. It is usually unilateral, and ophthalmic involvement may be noted as bluish and patchy discoloration of the sclera. Malignant degeneration (melanoma) is rarely reported, and therapy is quite difficult, although laser treatment may improve the appearance [43].

Nevus of Ito is a similar dermal melanocytosis seen in the supraclavicular, scapular, and deltoid regions.

\section{Café Au Lait Macules}

Café au lait macules are epidermal pigmented macules seen in $0.3 \%$ to $18 \%$ of neonates [3]. They present as light tan to brown, round, or oval macules and patches and can occur anywhere on the body. Their size may vary from a few millimeters to several centimeters. Most often, café au lait macules are sporadic and are not associated with a syndrome. However, multiple lesions may be associated with neurofibromatosis (requires $\geq 6$ lesions measuring 
over $5 \mathrm{~mm}$ in the prepubertal child and accompanied by at least one other type 1 neurofibromatosis diagnostic feature), McCune-Albright syndrome (usually large unilateral lesions with a sharp respect for the midline, in association with precocious puberty and bony fibrous dysplasia), and other rare disorders [44].

\section{Disorders Presenting With Skin Erythema and Scaling}

There are several disorders which may present with skin erythema and scaling (Table 2). A few of these disorders are discussed here.

\section{Atopic Dermatitis}

Atopic dermatitis (AD) is an extremely common skin disorder, affecting an estimated 17\% of all children in the United States [45]. Children with AD often have an atopic familial predisposition, as well as an increased risk for other atopic disorders including allergic rhinoconjunctivitis, asthma, and food allergy. Atopic dermatitis is often the initial manifestation of the "atopic march" to these other atopic diseases [46].

In neonates and infants, AD presents as scaly, erythematous patches and plaques of the extensor surfaces of the extremities, the trunk and the face (especially cheeks). Oozing and crusting may be present. When facial involvement is diffuse, the nose and perinasal areas are often spared, a pathognomonic finding termed the "headlight sign." The diaper area is characteristically spared, and pruritus is a consistent feature. Scalp involvement may be present, and the areolae may be edematous, scaly, and crusted. It may be difficult to distinguish seborrheic dermatitis from atopic dermatitis in the infant; however, involvement of the diaper and umbilical areas favor the former. Characteristic antecubital and popliteal accentuation of $\mathrm{AD}$ is seen later, usually during the toddler years $[46,47]$.

Secondary superinfection with $S$ aureus is very common in AD and usually presents with pustules and crusts (although the classic "honey color" is often lacking). Other infections which may occur with increased frequency include herpes simplex virus ("eczema herpeticum"), molluscum contagiosum, and warts. The management of neonatal/infantile AD includes brief daily baths, emolliation, topical corticosteroids (usually low-potency, occasionally mid-potency for more severe nonfacial, nonfold areas), and oral antihistaminic agents for control of pruritus. Secondary infection, when present, should be treated as well $[46,47]$.

\section{Collodion Baby/lchthyoses}

The term collodion baby refers to a newborn who is born encased in a shiny membrane that covers the entire skin surface. Its presence may result in flexion contractures,

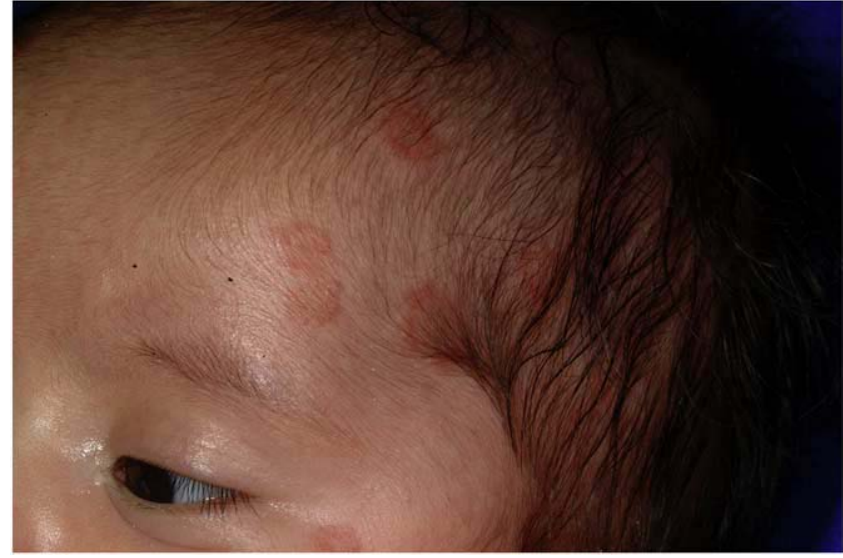

Figure 9 Neonatal lupus erythematosus. Erythematous, slightly scaly, annular plaques on sun-exposed areas of the face and scalp. Early recognition of this condition is vital, given the potential association with congenital heart block.

eclabium, ectropion, and distortion of the ear helices. This presentation may occur in association with a variety of disorders. The membrane is gradually shed over days to weeks but should be allowed to do so spontaneously in the setting of a humidity-controlled isolette. Potential complications for collodion babies include respiratory distress (secondary to chest restriction), fluid loss, electrolyte imbalances, and temperature instability. The majority of collodion babies turn out to have a form of ichthyosis termed nonbullous congenital ichthyosiform erythroderma. Other disorders which may present as a collodion baby include lamellar ichthyosis, X-linked recessive ichthyosis, Netherton syndrome, ectodermal dysplasia, and Gaucher disease. Occasionally, the skin appears and remains normal after shedding of a collodion membrane [48].

\section{Neonatal Lupus Erythematosus}

Neonatal lupus erythematosus (NLE) is an acquired disorder caused by transplacental transfer of maternal antibodies against the RNA proteins (Ro/SS-A and La/SS-B). A minority of affected infants have antibodies against U1 ribonucleoprotein. The risk of infants having NLE is $1 \%$ to $20 \%$ in mothers with anti Ro/La antibodies $[49,50]$. The skin is involved in approximately $50 \%$ of infants, and these changes may be present at birth or develop within the first few weeks of life $[49,50]$.

Neonatal lupus erythematosus presents with erythematous, slightly scaly patches, which may also reveal subtle atrophy and/or telangiectasia. The patches may be annular (ie, have central clearing) and prominent involvement of the periorbital areas may lead to the appearance of "raccoon eyes" (Figure 9). The face, head and neck are most commonly involved. The importance of prompt recognition of NLE is its association with irreversible congenital heart block, of which it is the most common 
cause. Transient liver involvement and hematologic cytopenias may also occur [51]. Mothers of infants with NLE have an increased risk of systemic lupus erythematosus, Sjogren syndrome, and mixed connective tissue disease, as well as a recurrence risk of $25 \%$ for NLE (especially the heart block) in future pregnancies [52].

\section{When To Worry About Immunodeficiency}

Skin eruptions may be the presenting feature for various forms of immunodeficiency. In this setting, patients may present with atopic- or seborrheic-like dermatitis, erythroderma, and/or severe intertrigo (redness in the fold areas). In addition, cutaneous infections may also point to an underlying immune defect, especially those that are severe or which are caused by unusual organisms. Clues to underlying immunodeficiency include severe and recalcitrant skin eruptions, poor response to therapy, cutaneous graft versus host disease on histologic examination (when skin biopsy is performed), and recurrent infection. In addition, growth failure, diarrhea, recurrent sinopulmonary infections, and alopecia may be other salient features.

\section{References}

1. Wagner A. Distinguishing vesicular and pustular disorders in the neonate. Curr Opin Pediatr 1997;9:396-405.

2. Gilliam AE, Pauporte M, Frieden IJ. Vesiculobullous and erosive diseases in the newborn. In: Bolognia JL, Jorizzo JL, Rapini RP, editors. Dermatology. 2nd ed. New York, NY: Elsevier; 2008. p. 475-8.

3. Conlon JD, Drolet BA. Skin lesions in the neonate. Pediatr Clin North Am 2004;51:863-88.

4. Nanda S, Reddy BS, Ramji S, et al. Analytical study of pustular eruptions in neonates. Pediatr Dermatol 2002;19:210-5.

5. Van Praag MC, Van Rooij RW, Folkers E, et al. Diagnosis and treatment of pustular disorders in the neonate. Pediatr Dermatol 1997;14:131-3.

6. Niamba P, Weill FX, Sarlangue J, et al. Is common neonatal cephalic pustulosis (neonatal acne) triggered by Malassezia sympodialis? Arch Dermatol 1998;134:995-8.

7. Johr RH, Schachner LA. Neonatal dermatologic challenges. Pediatr Rev 1997;18:86-94.

8. Smolinski KN, Shah SS, Honig PJ, et al. Neonatal cutaneous fungal infections. Curr Opin Pediatr 2005;17:486-93.

9. Mancini AJ, Frieden IJ, Paller AS. Infantile acropustulosis revisited: history of scabies and response to topical corticosteroids. Pediatr Dermatol 1998;15:337-41.

10. Martin JM, Green M. Group A streptococcus. Semin Pediatr Infect Dis 2006;17:140-8.

11. Sandhu K, Kanwar AJ. Generalized bullous impetigo in a neonate. Pediatr Dermatol 2004;21:667-9.

12. Stanley JR, Amagai M. Pemphigus, bullous impetigo, and the staphylococcal scalded-skin syndrome. N Engl J Med 2006;355: 1800-10.

13. Gupta R, Warren T, Wald A. Genital herpes. Lancet 2007;370:2127-37.

14. Gardella C, Handsfield HH, Whitley R. Neonatal herpes-the forgotten perinatal infection. Sex Transm Dis 2008;35:22-4.

15. Brown ZA, Vontver LA, Benedetti J, et al. Effects on infants of a first episode of genital herpes during pregnancy. N Engl J Med 1987;317: 1246-51.

16. Prober CG, Sullender WM, Yasukawa LL, et al. Low risk of herpes simplex virus infections in neonates exposed to the virus at the time of vaginal delivery to mothers with recurrent genital herpes simplex virus infections. N Engl J Med 1987;316:240-4.

17. Handsfield HH, Waldo AB, Brown ZA, et al. Neonatal herpes should be a reportable disease. Sex Transm Dis 2005;32:521-5.

18. Friedlander SF, Bradley JS. Viral infections. In: Eichenfield L, Frieden IL, Esterly NB, editors. Neonatal Dermatology. 2nd ed. London: Saunders Elsevier; 2008. p. 195.

19. Pouletty P, Chomel JJ, Thouvenot D, et al. Detection of herpes simplex virus in direct specimens by immunofluorescence assay using a monoclonal antibody. J Clin Microbiol 1987;25:958-9.

20. Kimberlin DW, Lin CY, Jacobs RF, et al. Safety and efficacy of highdose intravenous acyclovir in the management of neonatal herpes simplex virus infections. Pediatrics 2001;108:230-8.

21. Hernandez-Machin B, Reyes CS, Vargas MM, et al. Picture of the month: congenital cutaneous candidiasis. Arch Pediatr Adolesc Med 2007;161:907-8

22. Gibney MD, Siegfried EC. Cutaneous congenital candidiasis: a case report. Pediatr Dermatol 1995;12:359-63.

23. Darmstadt GL, Dinulos JG, Miller Z. Congenital cutaneous candidiasis: clinical presentation, pathogenesis, and management guidelines. Pediatrics 2000;105:438-44.

24. Aldana-Valenzuela C, Morales-Marquec M, Castellanos-Martínez J et al. Congenital candidiasis: a rare and unpredictable disease J Perinatol 2005;25:680-2.

25. Rowen JL, Atkins JT, Levy ML, et al. Invasive fungal dermatitis in the $<$ or $=1000$-gram neonate. Pediatrics 1995;95:682-7.

26. Heukelbach J, Feldmeier H. Scabies. Lancet 2006;367:1767-74.

27. Yamasaki O, Yamaguchi T, Sugai M, et al. Clinical manifestations of staphylococcal scalded-skin syndrome depend on serotypes of exfoliative toxins. J Clin Microbiol 2005;43:1890-3.

28. Faden H. Neonatal staphylococcal skin infections. Pediatr Infect Dis J 2003;22:389.

29. Nishifuji K, Sugai M, Amagai M. Staphylococcal exfoliative toxins: "molecular scissors" of bacteria that attack the cutaneous defense barrier in mammals. J Dermatol Sci 2008;49:21-31.

30. El Helali N, Carbonne A, Naas T, et al. Nosocomial outbreak of staphylococcal scalded skin syndrome in neonates: epidemiological investigation and control. J Hosp Infect 2005;61:130-8.

31. McGrath JA, Mellerio JE. Epidermolysis bullosa. Br J Hosp Med (Lond) 2006;67:188-91.

32. Bruckner AL. Epidermolysis bullosa. In: Eichenfield L, Frieden IL, Esterly NB, editors. Neonatal dermatology. 2nd ed. London: Saunders Elsevier; 2008. p. 159-73.

33. Uitto J, Richard G. Progress in epidermolysis bullosa: genetic classification and clinical implications. Am J Med Genet 2004;131: 61-74.

34. Fine JD, Eady RAJ, Bauer EA, et al. Revised classification system for inherited epidermolysis bullosa: report of the second International Consensus meeting on diagnosis and classification of epidermolysis bullosa. J Am Acad Dermatol 2000;42:1051-2.

35. Uitto J. Molecular diagnostics of epidermolysis bullosa: novel pathomechanisms and surprising genetics. Exp Dermatol 1999;8: 92-5.

36. Bello YM, Falabella AF, Schachner LA. Management of epidermolysis bullosa in infants and children. Clin Dermatol 2003;21:278-82.

37. Bruckner AL, Frieden IJ. Hemangiomas of infancy. J Am Acad Dermatol 2003;48:477-93.

38. Drolet BA, Esterly NB, Frieden IJ. Hemangiomas in children. N Engl J Med 1999;341:173-81.

39. Frieden IJ, Reese V, Cohen D. PHACE syndrome. The association of posterior fossa brain malformations, hemangiomas, arterial anomalies, coarctation of the aorta and cardiac defects, and eye abnormalities. Arch Dermatol 1996;132:307-11.

40. Mancini AJ. Vascular disorders of infancy and childhood. In: Paller AS, Mancini AJ, editors. Hurwitz Clinical Pediatric Dermatology. 3rd ed. London: Elsevier; 2006. p. 322-31. 
41. Ochiai T, Suzuki Y, Kato T, et al. Natural history of extensive Mongolian spots in mucopolysaccharidosis type II (Hunter syndrome): a survey among 52 Japanese patients. J Eur Acad Dermatol Venereol 2007;21:1082-5.

42. Mendez HM, Pinto LI, Paskulin GA, Ricachnevsky N. Is there a relationship between inborn errors of metabolism and extensive Mongolian spots? Am J Med Genet 1993;47:456-7.

43. Taieb A, Boralevi F. Hypermelanoses of the newborn and of the infant. Dermatol Clin 2007;25:327-36.

44. Korf BR. Diagnostic outcome in children with multiple café au lait spots. Pediatrics 1992;90:924-7.

45. Laughter D, Istvan JA, Tofte SJ, et al. The prevalence of atopic dermatitis in Oregon schoolchildren. J Am Acad Dermatol 2000;43:649-55.

46. Spergel JM, Paller AS. Atopic dermatitis and the atopic march. J Allergy Clin Immunol 2003;112(6 Suppl):S118-27.
47. Eigenmann PA. Clinical features and diagnostic criteria of atopic dermatitis in relation to age. Pediatr Allergy Immunol 2001;14 (12Suppl):69-74.

48. Van Gysel D, Lijnen RL, Moekti SS, et al. Collodion baby: a follow-up study of 17 cases. J Eur Acad Dermatol Venereol 2002; $16: 472-3$.

49. Lee LA. Neonatal lupus erythematosus. J Invest Dermatol 1993;100: 9S-13S.

50. Silverman ED, Laxer RM. Neonatal lupus erythematosus. Rheum Dis Clin North Am 1997;23:599-618.

51. Lee LA. Neonatal lupus: clinical features and management. Pediatr Drugs 2004;6:71-8.

52. McCune AB, Weston WL, Lee LA. Maternal and fetal outcome in neonatal lupus erythematosus. Ann Intern Med 1987;106: 518-23. 